\title{
Analysis on Conveying of Miniature and Microparts on a Platform Sub- jected to Sinusoidal Displacement Cycles with Controlled Dry Friction
}

\author{
Sigitas KILIKEVIČIUS*, Algimantas FEDARAVIČIUS**, Virginija DAUKANTIENE****, \\ Kristina LIUTKAUSKIENE $* * * *$ \\ *Kaunas University of Technology, Studentu 56, 51424 Kaunas, Lithuania, E-mail: sigitas.kilikevicius@ktu.lt \\ **Kaunas University of Technology, Studentu 56, 51424 Kaunas, Lithuania, E-mail: algimantas.fedaravicius@ktu.lt \\ ***Kaunas University of Technology, Studentu 56, 51424 Kaunas, Lithuania, E-mail: virginija.daukantiene@ktu.lt \\ ****Kaunas University of Technology, Studentu 56, 51424 Kaunas, Lithuania, E-mail: kristina.liutkauskiene@ktu.lt \\ crossref http://dx.doi.org/10.5755/j02.mech.28195
}

\section{Introduction}

The trend toward miniaturisation of mass-produced products motivates the development of microtechnologies and related science fields. Many methods for micropart conveying are being developed, researched, and applied in practice. Microparts can be directionally displaced by various means ranging from acoustic methods suited for manipulations of large clusters of microparticles to single pick and place operations.

Near-field acoustic manipulation suitable to operate with micro and nanoparticles in a confined evanescent Bessel beam was proposed by Gires and Poulain [1]. Xu et al. [2] analysed acoustic manipulation of microparticles in a cylindrical cavity using the acoustic radiation force. Ostasevicius et al. [3] applied an acoustic field for separation and purification of a large volume of microparticles dispersed in a biological suspension poured into a conical fluid container. Due to the acoustic excitation, the suspension was pushed upwards and the microparticles accumulated in the nodal zones of the resonating container walls by purifying the suspension that accumulates in the anti-nodal zones. Kashima et al. [4] analysed two-dimensional conveying of microparts in air using flexural vibration of a plate with four ultrasonic transducers and a reflector. When a part is considerably smaller than the wavelength of the acoustic standing wave in air, the part is trapped along the horizontal nodal planes. They showed that, the flexural standing wave on the plate can be displaced by controlling the phase difference of the two pairs of the transducers, meanwhile the acoustic standing wave position in air can be shifted in the same direction. This technique allows the trapped microparts to be conveyed.

Xiao et al. [5] investigated a large stroke manipulator for micro/nano manipulation on a plane. By using two electromagnetic actuators for each axis, the micromanipulator operated in planar spiral and quadrant motions. A spatial flexible device for micropart translation based on flexure hinges, which can perform the translational motion along the $\mathrm{Z}$ axis as well as rotations around $\mathrm{X}$ and $\mathrm{Y}$ axes was developed by Zhao et al. [6]. Lienemann et al. [7] analysed conveying realised through a micro fluidic wetting-based approach by applying the many-body dissipative particle dynamics method. A programmable platform with conductive electrodes was used by Kritikou et al. [8] for the displacement of microparts influenced by electrostatic forces. Yuan et al. [9] formulated nonprehensile manipulation planning as a reinforcement learning problem using a trained deep Q-network to generate actions based on the learned policy. In comparison to a human subject, they concluded that the network has achieved comparable performance to the human. They also qualitatively showed that the network is reactive and adaptive to uncertainties, such as the sudden changes of objects and target positions, low-friction coefficients and distraction objects. Ta and Cheah [10] proposed the first stochastic control technique for the purpose of the simultaneous orientation and transportation of micro-objects with Brownian perturbations. The micro-particles were utilized as fingertips to first grasp a target micro-object, and they were optically trapped and driven by laser beams [10]. Piatkowski et al. [11] analysed the angular positioning process of cuboidal objects with the use the system of two oblique friction forces fields created by conveyor belts. Chen et al. studied [12] the mechanism of particle directional conveyance in a trough with finlike asperities under longitudinal vibrations by applying numerical simulations based on the discrete element method. They determined that the asymmetric force induced by the finlike asperities and its cumulative effect over time influenced the particle directional conveyance. Rizwan and Shiakolas [13-15] developed a conveyor for microparts based on controlled deformation of a flexible continuous surface. The sensitivity of micropart motion and micropositioning as a function of micropart and surface material properties and input actuator properties were studied. They highlighted that the motion of the micropart depends on the relative surface roughness, surface deformation profile, the dynamic compression of asperities and their effect on the dynamic friction coefficient based on extending Kogut-Etsion friction model through a quasi-dynamic coefficient of friction estimation [14]. Langford et al. [16] investigated the effect of two-dimensional friction anisotropy of micro pillared surfaces on the conveying parameters of microparts. The system for conveying microparts was composed of surface of oriented micro-pillars inspired by natural cilia structures along with a vertical vibration source. It was confirmed that the velocity of the micropart was directly proportional to both the frequency and the amplitude of the vibratory excitation [16].

A nonprehensile manipulation technique was proposed where the required dynamic directionality was achieved via a system asymmetry that was induced by periodic alteration of the effective coefficient of dry friction between the part and the manipulation platform in [17-19].

This paper analyses the conveying process of miniature and microparts on a manipulation platform subjected to sinusoidal displacement cycles in the horizontal direction 
when the coefficient of dry friction between the part and the platform is controlled. Technically, the coefficient of dry friction can be altered dynamically by utilising high frequency vibrations [20-25]. The aim of the analysis is to determine the influence of frictional properties, friction control and sinusoidal excitation parameters on the characteristics of the conveying process.

\section{Modelling of conveying with dry friction control}

\subsection{Dynamics of conveying with dry friction control}

The dynamic model of part conveying with dry friction control in respect of sinusoidal displacement cycles is shown in Fig. 1.

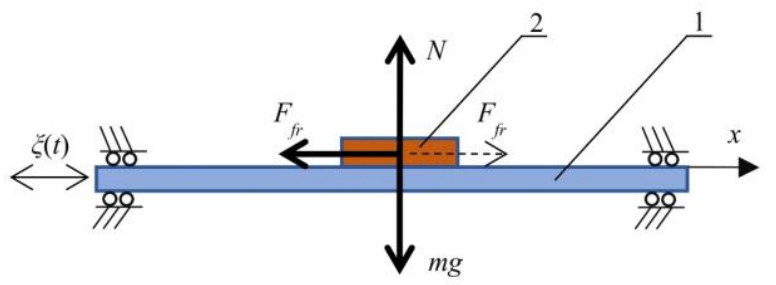

Fig. 1 The dynamic model of part conveying with dry friction control in respect of sinusoidal displacement cycles: 1 - platform with a possibility to control dry friction; 2 - part for conveyance

The conveying system consists of a horizontal platform excited by sinusoidal displacement cycles:

$$
\xi(t)=A \sin \omega t,
$$

where: $t$ is time; $A$ is the amplitude of displacement; $\omega$ is the angular frequency.

The equation of the relative part motion on the platform is as follows:

$$
m \ddot{x}-m A \omega^{2} \sin \omega t+F_{f r}=0
$$

where: $m$ is the mass of the micropart; $F_{f r}$ is the dry friction force. Applying the Dahl dynamic friction model [26, 27], the dry friction force can be expressed using the following equations:

$$
\left\{\begin{array}{l}
F_{f r}=\sigma_{0} z, \\
\dot{z}=\left(1-\frac{\sigma_{0}}{\mu(\tau) m g} z \operatorname{sgn}(\dot{x})\right) \dot{x},
\end{array}\right.
$$

where: $\tau=\omega t, \sigma_{0}$ is the stiffness of contact surface asperities; $z$ is the state variable interpreted as elastic displacement of the asperities; $\mu(\tau)$ is the effective dry friction coefficient, which is not constant. With respect of sinusoidal displacement cycles, the dry friction is controlled in the manner presented in Fig. 2. In each of the period of the sinusoidal excitation, the dry friction is being reduced to the $\mu_{2}$ value at a phase angle of $\tau_{1}$, and, at a phase angle of $\tau_{2}$, it is being changed back to its nominal value $\mu_{1}$ (Fig. 2). Therefore, in each period of the sinusoidal excitation, the dry friction coefficient is reduced for a fraction, which is expressed as $\Delta \tau=\tau_{2}-\tau_{1}$. The parameter $\varphi$ shown in Fig. 2 is the phase shift between the function of the effective dry friction coefficient $\mu(\tau)$ and the sinusoidal displacement cycles $\xi(\tau)$.

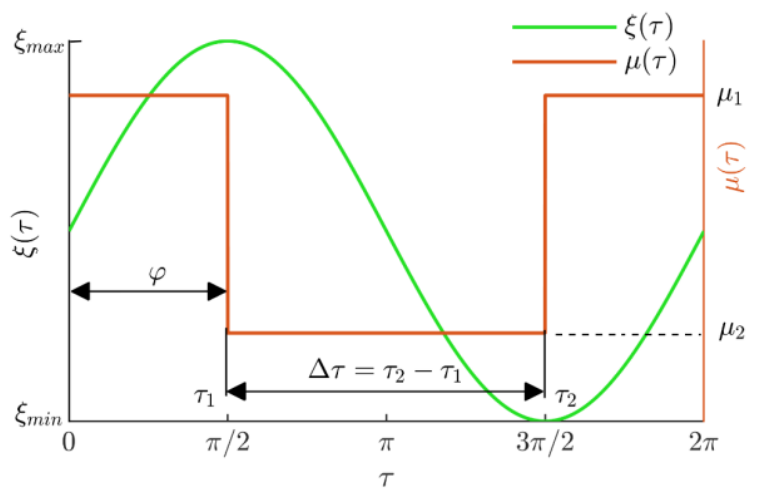

Fig. 2 Dry friction control in respect of the sinusoidal displacement period

Such a way of dry friction control eliminates the symmetry of the mechanical system. Due to this, a directional motion of the micropart is achieved since net frictional forces are created in each sinusoidal displacement cycle of the platform. The performance of the conveying process depends on how high the asymmetry is.

In order to analyse the influence of various system's parameters on the characteristics of the conveying process, a modelling of conveying was carried out. The ode15s solver for stiff differential equations was applied in MATLAB to solve the equation of the relative part motion numerically.

The following values were used for the modelling of the micropart conveying process: $A=1.6 \mathrm{~mm}$, $\omega=125.7 \mathrm{rad} / \mathrm{s} m=2.4 \cdot 10^{-5} \mathrm{~kg}, \varphi=\pi / 2, \Delta \tau=\pi, \mu_{1}=0.1$, $\mu_{1} / \mu_{2}=8 . \sigma_{0}=10^{5} \mathrm{~N} / \mathrm{m}$. The value of $\sigma_{0}$ was selected based on the value reported in published research articles [28-30].

\subsection{Modelling results}

The modelling of the conveying process with controlled dry friction clearly demonstrated that the direction of conveying can be easily controlled by changing the phase shift between the function of the effective dry friction coefficient and the horizontal sinusoidal displacement cycles. Fig. 3, a demonstrates the influence of the phase shift $\varphi$ between the function of the effective dry friction coefficient and the horizontal sinusoidal cycles on the average stable conveying velocity $v$. Under the investigated parameters, the highest values of the average stable conveying velocity were observed at phase shift values around $\pi / 4$. The conveying direction is reversed when the phase shift is in a range approximately from $5 \pi / 6$ to $7 \pi / 6$. Under higher values of $\Delta \tau$, higher values of $\varphi$ are needed to reverse the direction of conveying.

The conveying velocity can be easily controlled in a wide range by changing $\Delta \tau$. Fig. 3 , b shows the influence of $\Delta \tau$ on the average stable conveying velocity. As $\Delta \tau$ increases, $v$ increases until, at some point, it reaches the maximum value and starts to decrease. This point depends on $\varphi$. At $\varphi=2 \pi / 9$, the maximum value of $v$ is obtained when the value of $\Delta \tau$ is around $3 \pi / 2$. At $\varphi=4 \pi / 9$, the maximum is obtained when $\Delta \tau$ is around $10 \pi / 9$, while at $\varphi=2 \pi / 3$, the maximum is obtained when $\Delta \tau$ is close to $8 \pi / 9$. 
The influence of frictional properties was determined using the developed mathematical model. The modelling showed that the average conveying velocity tends to decrease as the nominal dry friction coefficient increases (Fig. 3, c). It was also demonstrated that the average conveying velocity increases with an increase in the ratio of friction reduction $\mu_{1} / \mu_{2}$ (Fig. 3, d).

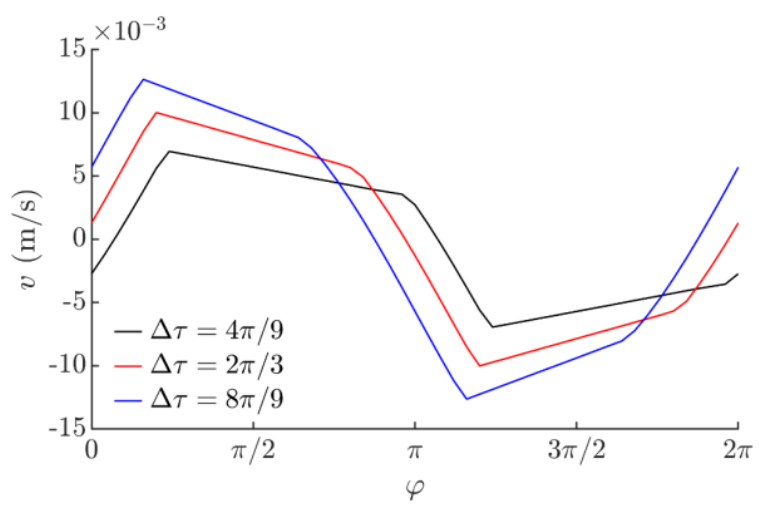

a

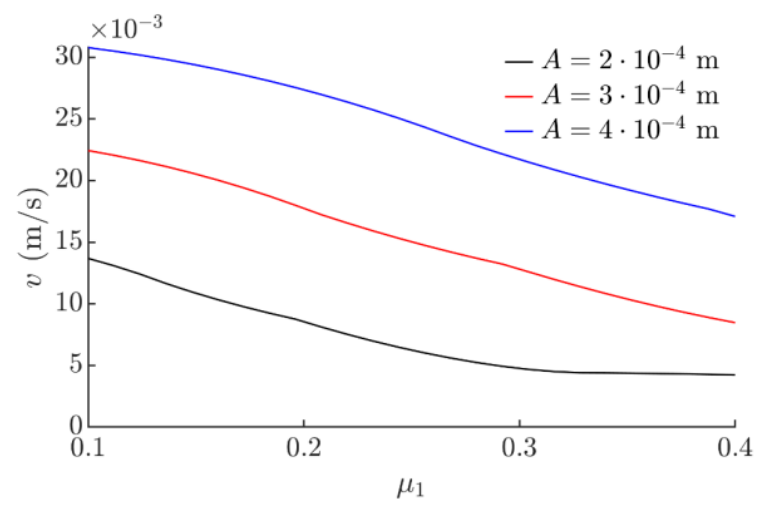

c
The modelling demonstrated that the mass of the micropart $m$ does not have a significant influence on the average conveying velocity in a range of $m$ up to approximately $10^{-3} \mathrm{~kg}$ (Fig. 3, e). In a range of $m$ values that were higher than $10^{-3} \mathrm{~kg}$, a more significant influence of $m$ on $v$ was observed. In this range, the average conveying velocity increases with an increase in the mass of the micropart to be conveyed (Fig. 3, e).

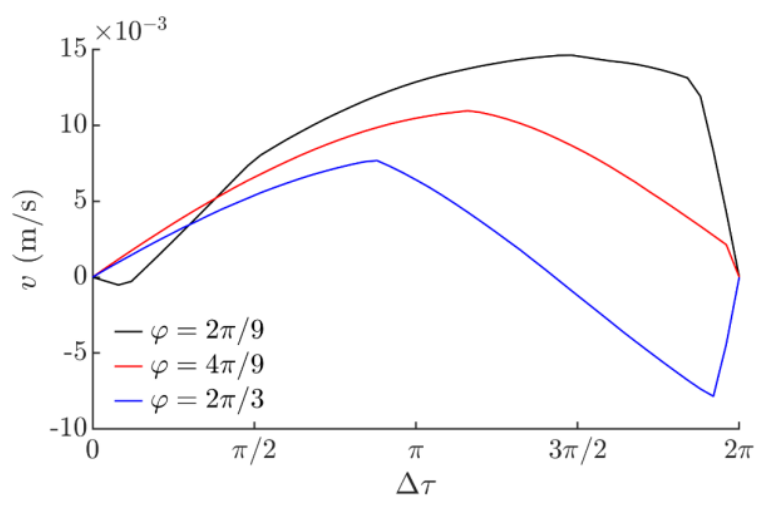

b

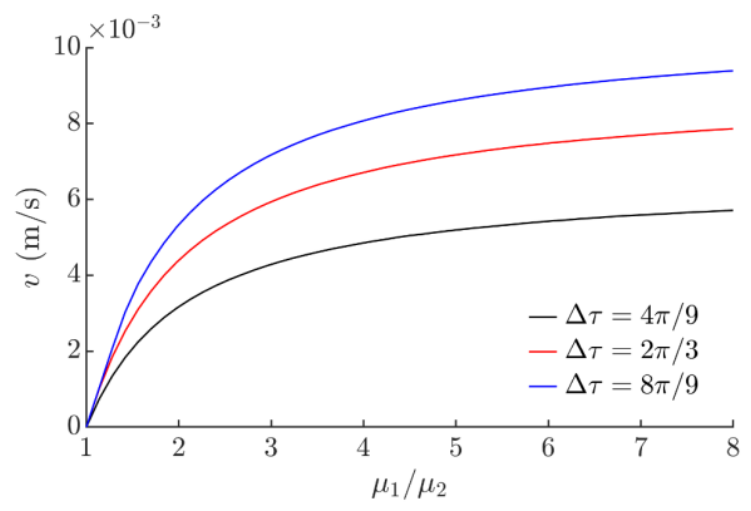

$\mathrm{d}$

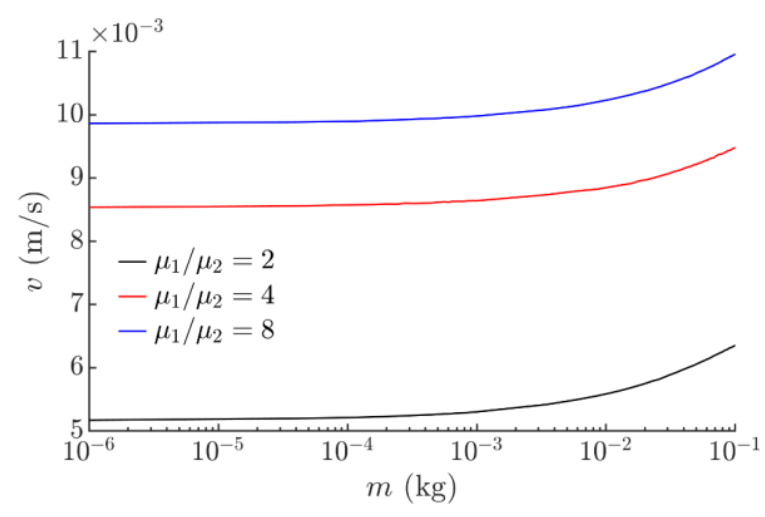

e

Fig. 3 Average stable conveying velocity depending on: a) the phase shift; b) $\Delta \tau$; c) the nominal dry friction coefficient; d) the ratio of friction reduction; e) the mass of the micropart to be conveyed

The capability to control the conveying velocity and the motion direction is clearly seen from the three-dimensional representation of the average stable conveying velocity $v$ as a function of $\varphi$ and $\Delta \tau$ (Fig. 4 , a). The threedimensional representation of the average stable conveying velocity as a function of the nominal friction coefficient and the effective friction coefficient reduction is shown in Fig. 4, b. It shows how the average conveying velocity decreases when the nominal friction coefficient increases and the ratio $\mu_{1} / \mu_{2}$ decreases. Fig. 4 , c shows the three-dimensional representation of the average stable conveying velocity as a function of the nominal friction coefficient and the frequency of the sinusoidal excitation. It suggests that under lower values of sinusoidal amplitude $A$, the average conveying velocity is influenced more significantly by the nominal friction coefficient (Fig. 4, c). It is obvious that higher values of the amplitude (Fig. 4, c) and frequency (Fig. 4, d) of the sinusoidal excitation result in higher values of the average stable conveying velocity. 


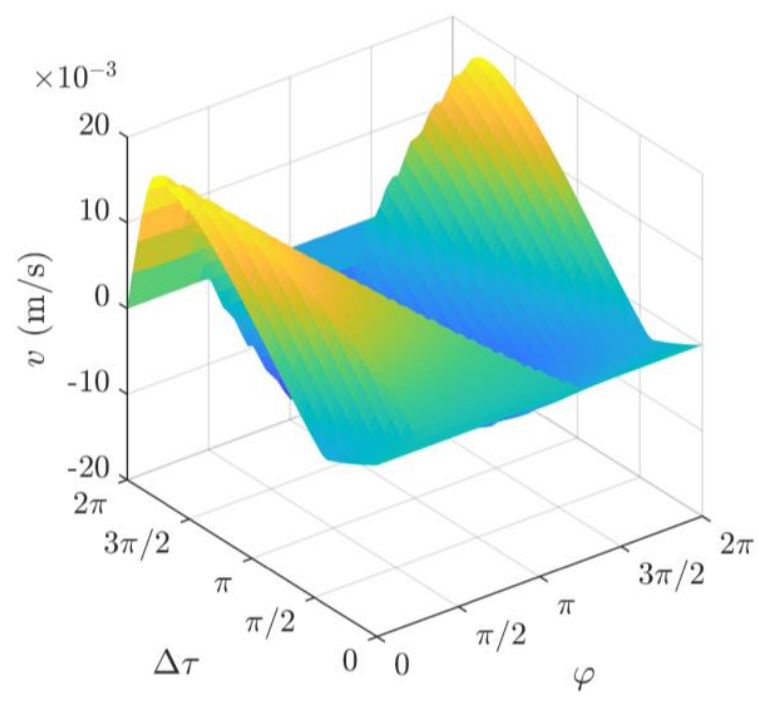

a

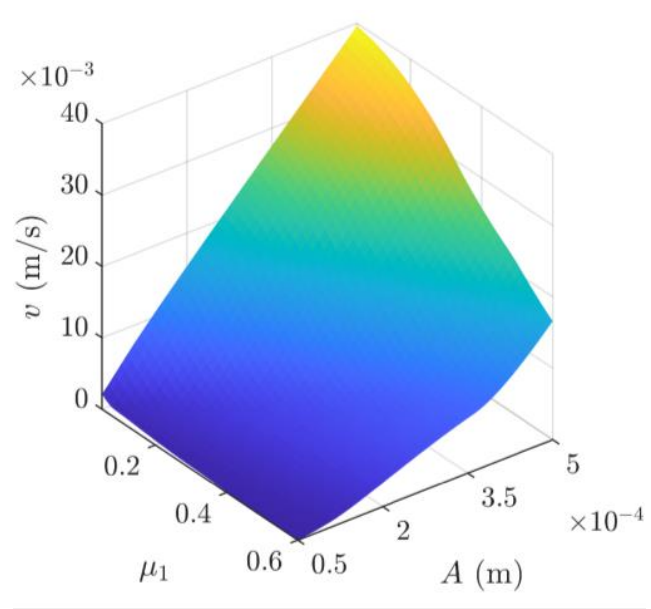

c

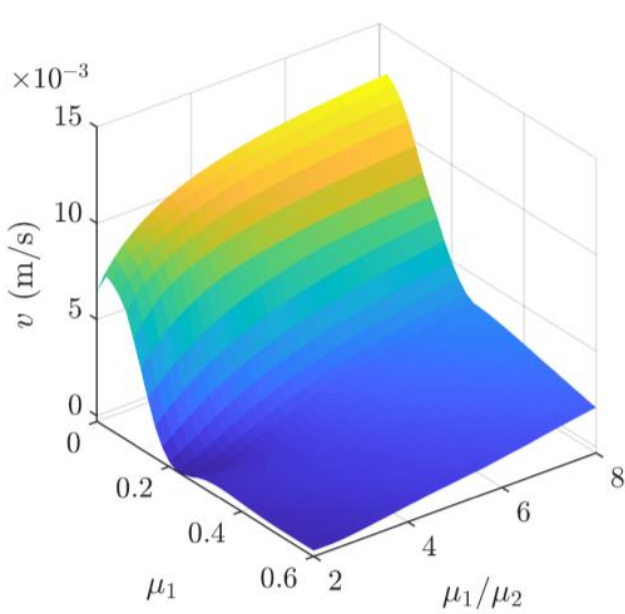

b

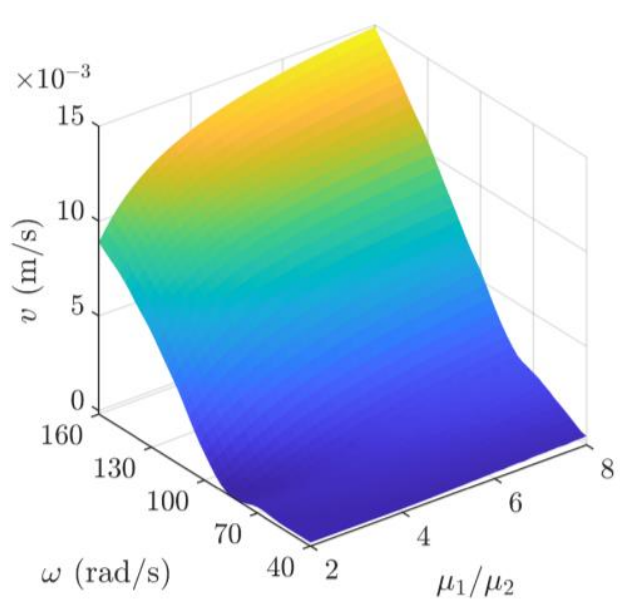

d

Fig. 4 Three-dimensional representation of the average stable conveying velocity as a function of: a) the phase shift and $\Delta \tau$, b) the nominal dry friction coefficient and the ratio of friction reduction; c) the nominal dry friction coefficient and the amplitude of sinusoidal displacement cycles; d) the angular frequency of sinusoidal displacement cycles and the ratio of friction reduction

\section{Experimental analysis}

\subsection{Methodology}

An experimental analysis was carried out to test the theoretical observations in practice. High frequency vibratory excitation was considered as a measure to control the dry friction dynamically.

The scheme of the experimental setup for the analysis of micropart conveying with controlled dry friction is presented in Fig. 5. The platform base 9 is subjected to sinusoidal displacement cycles by employing an electrodynamic shaker 1 . A piezoelectric actuator 10 is mounted on the platform base and it generates high-frequency vibrations to control the effective friction coefficient between the micropart 8 and the platform for conveying 11 . The signals of sinusoidal displacement cycles and high-frequency excitation for the piezoelectric actuator are generated by a RIGOL DG4202 arbitrary waveform generator 4 .

The signal for the piezoelectric actuator is composed of high frequency pulses in burst mode. This signal is synchronised in accordance with the period of sinusoidal displacement cycles. Similar like it is shown in Fig. 2, high frequency pulses are activated at the instant of time $\tau_{1}$ in respect of the period of the sinusoidal displacement cycles, and, at the instant of time $\tau_{2}$, high frequency pulses are deactivated. In this way, the piezoelectric actuator is active for a fraction equal to $\Delta \tau$ in each period of the sinusoidal displacement cycles of the platform with a phase shift of $\varphi$.

The signal of sinusoidal displacement cycles is amplified by an AMC iA4X125 amplifier 2, while the signal of high-frequency excitation for the piezoelectric actuator is amplified by an EPA-104 piezo linear amplifier 3. The vibrational response of the system is monitored by vibration sensors 6 and 7. All these signals from the sensors and the generator are monitored by a RIGOL DS1054 digital oscilloscope 5 .

General multilayer ceramic capacitors (MLCC) were chosen as microparts for conveying since they are widely used in microelectronics. The experimental setup was tested using $0402(1.0 \times 0.5 \times 0.5 \mathrm{~mm}), \quad 0805$ $(2.0 \times 1.25 \times 0.85 \mathrm{~mm})$ and $1206(3.2 \times 1.6 \times 0.85 \mathrm{~mm})$ type MLCC capacitors (Samsung) (Fig. 6). 


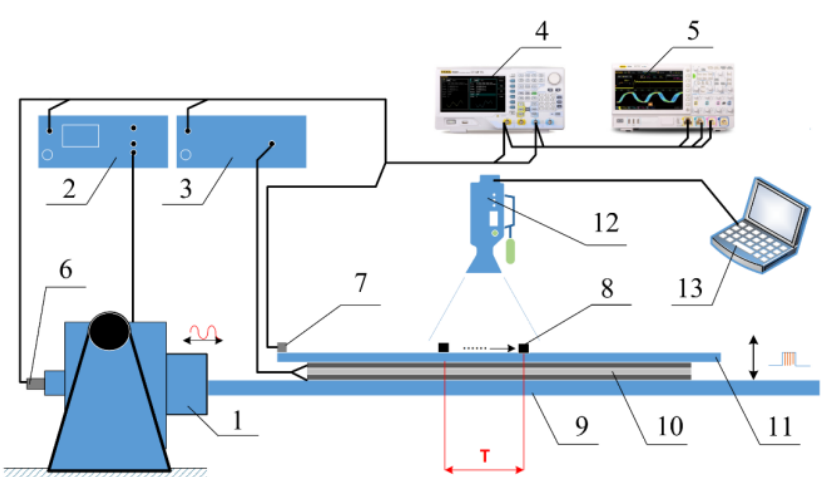

Fig. 5 Scheme of experimental setup: 1 -electrodynamic shaker; 2-low-frequency vibration amplifier; 3 - high-frequency vibration amplifier; 4 - arbitrary waveform generator; 5-digital oscilloscope; 6, 7 -vibration sensors; 8-micropart; 9-platform base subjected to sinusoidal displacement cycles; 10 - piezoelectric actuator; 11 - platform for conveying; 12 - camera; 13 - computer

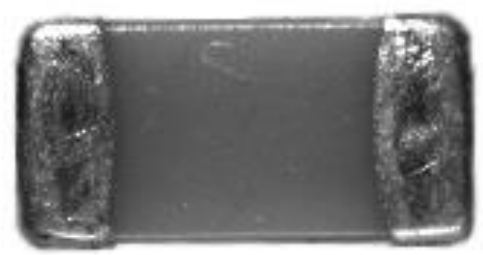

Fig. 6 Samsung 1210 MLCC capacitor

A Phantom v711 (1280×800 CMOS sensor, $1 \mathrm{Mpx}$, $20 \mu \mathrm{m}$ pixel size) high-speed camera 12 was used to track the conveying of the micropart. It was equipped with a Canon MP-E $65 \mathrm{~mm}$ f/2.8 1-5x Macro Lens. A frame rate of 500 frames per second at a resolution of $800 \times 800$ was selected for the recordings. A computer 13 was used to control the camera and store the recordings. In order to digitize the coordinates of the micropart over time, a video processing program was developed in MATLAB, employing the normalized cross-correlation approach.

\subsection{Experimental results}

Fig. 7 shows typical oscillograms of the signal for sinusoidal displacement cycles, the signal of high frequency pulses in burst mode for dry friction control, and the vibrational response of the platform. They reveal how the micro-

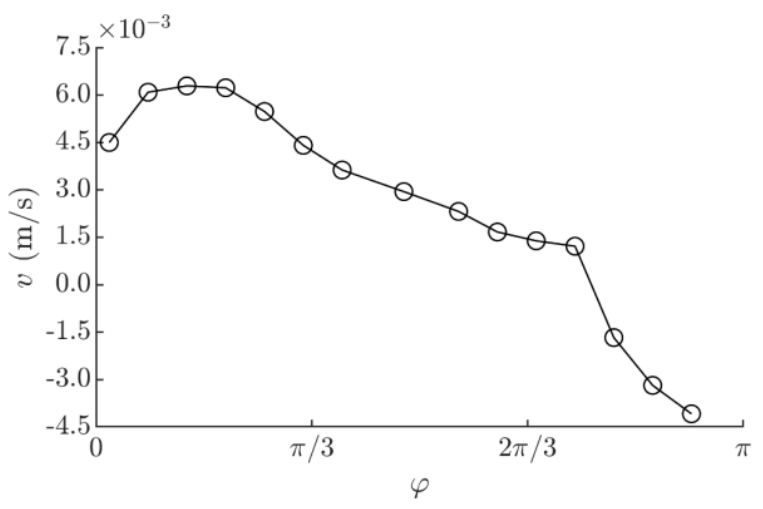

a part is being conveyed exactly according to the program of the control signals. Thus, it is possible to control the conveyance velocity, direction, and step size by adjusting the phase shift between the signal of high frequency pulses in burst mode and the signal of horizontal sinusoidal displacement cycles as well as the burst width which can be expressed as $\Delta \tau$.

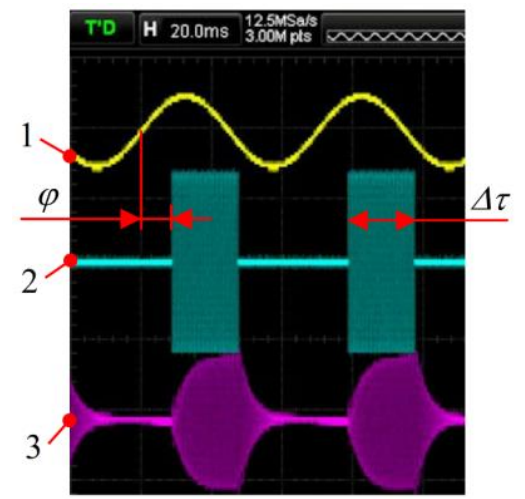

Fig. 7 Oscillograms of the system's signals: 1 - signal for sinusoidal displacement cycles; 2 - signal of high frequency pulses in burst mode for dry friction control; 3 - vibrational response of the platform

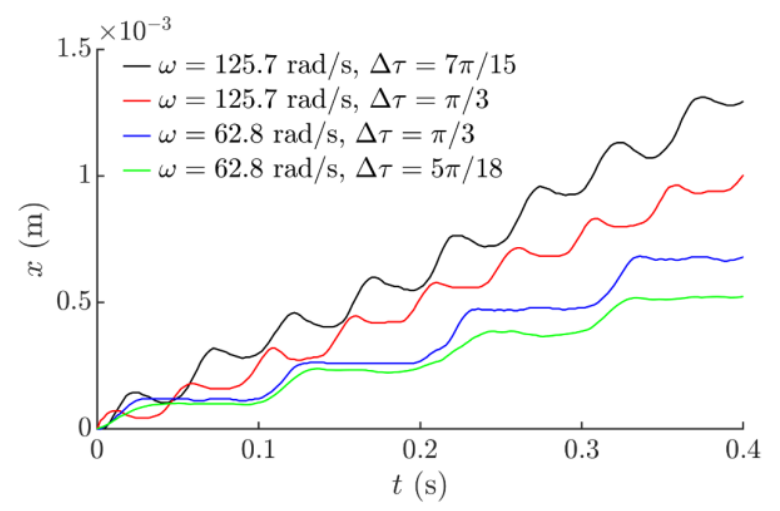

Fig. 8 Captured displacement of micropart over time at different $\omega$ and $\Delta \tau$ values, $A=0.16 \mathrm{~mm}, \varphi=\pi / 2$

The character of the displacement of micropart over time is shown in Fig. 8.

The moving micropart performs steps and the size of these steps depends on the control parameters (Fig. 8). A required step size can be maintained by adjusting the frequency and amplitude of sinusoidal excitation as well as $\Delta \tau$.

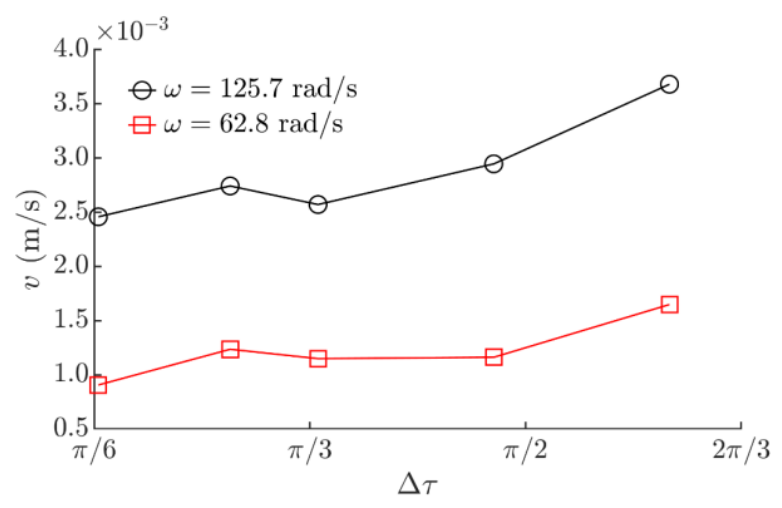

b

Fig. 9 Experimental average stable conveying velocity $v$ depending on: a) the phase shift $\varphi, A=0.16 \mathrm{~mm}, \omega=125.7 \mathrm{rad} / \mathrm{s}$, $\Delta \tau=7 \pi / 15 ;$ b) $\Delta \tau, A=0.16 \mathrm{~mm}, \varphi=\pi / 2$ 
Consistently with the modelling results, the experiments confirmed that the velocity and direction of conveying depend on the phase shift $\varphi$ (Fig. 9, a). Similarly, like it was observed in the modelling results (Fig. 3, a), the conveying direction was changed when $\varphi$ was near $5 \pi / 6$ (Fig. 9, a). Fig. 9, b shows the influence of $\Delta \tau$ on $v$ when $A=0.16 \mathrm{~mm}$ and $\varphi=\pi / 2$. As it was observed in the modelling results (Fig. 3, b), the experimentally obtained average stable conveying velocity was tending to increase with an increase in $\Delta \tau$ under the analysed conditions (Fig. 9, b). The experimental findings qualitatively confirmed the trends observed by the modelling and showed that the proposed method can be applied in practice.

\section{Conclusions}

A mathematical model of conveying process was developed applying the Dahl dynamic friction model, and it was solved numerically to determine the influence of frictional properties, friction control and sinusoidal excitation parameters on the characteristics of the conveying process. The modelling showed that the direction and velocity of conveying can be controlled by changing the phase shift between the function of the effective dry friction coefficient and the horizontal sinusoidal displacement cycles. The conveying velocity can be easily controlled in a wide range by changing $\Delta \tau$. The modelling showed that the average conveying velocity tends to decrease as the nominal dry friction coefficient increases. The average conveying velocity increases with an increase in the ratio of friction reduction. An increase in the amplitude or the frequency of the sinusoidal excitation results in an increase in the average conveying velocity.

To test the theoretical observations in practice, an experimental setup for micropart conveying with controlled dry friction was created and build. Consistently with the modelling results, the experimental results demonstrated how the stable conveying velocity, direction and step size are controlled by regulating the parameters of the signals for sinusoidal displacement cycles and high frequency pulses in burst mode for dry friction control. The experimental findings qualitatively confirmed the trends observed by the modelling and showed that the proposed method can be applied in practice. The conveying method can be practically used in conveying, feeding, manipulation and assembly systems for miniature and microparts in the mechatronics, electronic and other industries.

\section{Acknowledgments}

This research was funded by a grant (No. S-MIP-19-64) from the Research Council of Lithuania.

\section{References}

1. Gires, P.; Poulain, C. 2019. Near-field acoustic manipulation in a confined evanescent Bessel beam, Communications Physics 2(1): 1-8. http://dx.doi.org/10.1038/s42005-019-0191-z.

2. Xu, D.; Cai, F.; Chen, M.; Li, F.; Wang, C.; Meng, L., et al. 2019. Acoustic manipulation of particles in a cylindrical cavity: theoretical and experimental study on the effects of boundary conditions, Ultrasonics 93: 1825. http://dx.doi.org/10.1016/j.ultras.2018.10.003.

3. Ostasevicius, V.; Jurenas, V.; Gaidys, R.; Golinka, I.; Kizauskiene, L.; Mikuckyte, S. 2020. Development of a piezoelectric actuator for separation and purification of biological microparticles, Actuators 9(3): 61-74. http://dx.doi.org/10.3390/act9030061.

4. Kashima, R.; Koyama, D.; Matsukawa, M. 2015. Two-dimensional noncontact transportation of small objects in air using flexural vibration of a plate, IEEE Transactions on Ultrasonics, Ferroelectrics, and Frequency Control 62(12): 2161-2168. http://dx.doi.org/10.1109/TUFFC.2015.006998.

5. Xiao, X.; Li, Y.; Xiao, S. 2017. Development of a novel large stroke 2-DOF micromanipulator for micro/nano manipulation, Microsystem Technologies 23(7): 29933003. http://dx.doi.org/10.1007/s00542-016-2991-3.

6. Zhao, J.; Li, Y.; Xu, H. Design and analysis of a new type of spatial flexible micromanipulation platform. Proceedings of the 13th World Congress on Intelligent Control and Automation (WCICA): 199-204. http://dx.doi.org/10.1109/WCICA.2018.8630732.

7. Lienemann, J.; Weiß, D.; Greiner, A.; Kauzlaric, D.; Grünert, O.; Korvink, J. G. 2012. Insight into the micro scale dynamics of a micro fluidic wetting-based conveying system by particle based simulation, Microsystem Technologies 18(4): 523-530.

http://dx.doi.org/10.1007/s00542-012-1460-x.

8. Kritikou, G.; Aspragathos, N.; Moulianitis, V. 2019. Algorithms for the motion of randomly positioned hexagonal and square microparts on a "Smart platform" with electrostatic forces and a new method for their simultaneous centralization and alignment, Micromachines 10(12): 874. http://dx.doi.org/10.3390/mi10120874

9. Yuan, W.; Stork, J. A.; Kragic, D.; Wang, M. Y.; Hang, K. 2018. Rearrangement with nonprehensile manipulation using deep reinforcement learning, 2018 IEEE International Conference on Robotics and Automation (ICRA): 270-277. http://dx.doi.org/10.1109/ICRA.2018.8462863.

10. Ta, Q. M.; Cheah, C. C. 2019. Stochastic control for orientation and transportation of microscopic objects using multiple optically driven robotic fingertips, IEEE Transactions on Robotics 35(4): 861-872. http://dx.doi.org/10.1109/TRO.2019.2902064.

11. Piatkowski, T.; Wolski, M.; Dylag, K. 2019. Angular positioning of the objects by the system of two oblique friction force fields, Mechanism and Machine Theory 140: 668-685.

http://dx.doi.org/10.1016/j.mechmachtheory.2019.06.028.

12. Chen, H.; Jiang, S.; Liu, R.; Zhang, W. 2018. Particle directional conveyance under longitudinal vibration by considering the trough surface texture: Numerical simulation based on the discrete element method, Shock and Vibration 2018. http://dx.doi.org/10.1155/2018/8260462.

13. Rizwan, M.; Shiakolas, P. S. 2012. Towards the realization of a conveyor platform for microparts employing a deformable surface, Proceedings of the ASME 2012 International Mechanical Engineering Congress and Exposition: $311-318$. http://dx.doi.org/10.1115/IMECE2012-87910. 
14. Rizwan, M.; Shiakolas, P. S. 2012. Sensitivity analysis of micropart motion on a controlled deformable continuous surface, Proceedings of the ASME 2012 International Mechanical Engineering Congress and Exposition: 319-326. http://dx.doi.org/10.1115/IMECE2012-87922.

15. Rizwan, M. 2014. A one dimensional dry friction microconveyor platform: Modelling and analysis of micropart motion due to surface excitation.

16. Langford, W.; Ou, J., \& Yao, L. 2014. 2D friction anisotropy of MicroPillared surfaces for the translation of small objects.

17. Kilikevičius, S.; Liutkauskienė, K.; Fedaravičius, A. 2021. Nonprehensile manipulation of parts on a horizontal circularly oscillating platform with dynamic dry friction control, Sensors 21(16): 5581. http://dx.doi.org/10.3390/s21165581.

18. Kilikevičius, S.; Fedaravičius, A.; Daukantienė, V.; Liutkauskienė, K.; Paukštaitis, L. 2021. Manipulation of miniature and microminiature bodies on a harmonically oscillating platform by controlling dry friction, Micromachines 12(9): 1087. http://dx.doi.org/10.3390/mi12091087.

19. Kilikevičius, S.; Fedaravičius, A. 2021. Vibrational transportation on a platform subjected to sinusoidal displacement cycles employing dry friction control, Sensors 21(21): 7280 . http://dx.doi.org/10.3390/s21217280.

20. Popov, V. L.; Starcevic, J.; Filippov, A. E. 2010. Influence of ultrasonic in-plane oscillations on static and sliding friction and intrinsic length scale of dry friction processes, Tribology Letters 39(1): 25-30. http://dx.doi.org/10.1007/s11249-009-9531-6

21. Teidelt, E.; Starcevic, J.; Popov, V. L. 2012. Influence of ultrasonic oscillation on static and sliding friction, Tribology Letters 48(1): 51-62. http://dx.doi.org/10.1007/s11249-012-9937-4.

22. Gutowski, P.; Leus, M. 2012. The effect of longitudinal tangential vibrations on friction and driving forces in sliding motion, Tribology International 55: 108-118. http://dx.doi.o rg/10.1016/j.triboint.2012.05.023.

23. Capozza, R.; Vanossi, A.; Vezzani, A.; Zapperi, S. 2009. Suppression of friction by mechanical vibrations, Physical Review Letters 103(8): 085502. http://dx.doi.org/10.1103/PhysRevLett.103.085502.

24. Storck, H.; Littmann, W.; Wallaschek, J.; Mracek, M. 2002. The effect of friction reduction in presence of ultrasonic vibrations and its relevance to travelling wave ultrasonic motors, Ultrasonics 40(1): 379-383. http://dx.doi.org/10.1016/S0041-624X(02)00126-9.

25. Chowdhury, M. A.; Helali, M. 2008. The effect of amplitude of vibration on the coefficient of friction for different materials, Tribology International 41(4): 307-314. http://dx.doi.org/10.1016/j.triboint.2007.08.005.

26. Dahl, P. R. 1968. A solid friction model. A Solid Friction Model.

27. Dahl, P. R. 1976. Solid friction damping of mechanical vibrations, AIAA Journal 14(12): 1675-1682. http://dx.doi.org/10.2514/3.61511
28. Wang, P.; Ni, H.; Wang, R.; Li, Z.; Wang, Y. 2016. Experimental investigation of the effect of in-plane vibrations on friction for different materials, Tribology International 99: 237-247. http://dx.doi.org/10.1007/s11071-016-2999-3.

29. Pennestrì, E.; Rossi, V.; Salvini, P.; Valentini, P. P. 2016. Review and comparison of dry friction force models, Nonlinear Dynamics 83(4): 1785-1801. http://dx.doi.org/10.1007/s11071-015-2485-3.

30. Marques, F.; Flores, P.; Claro, J. P.; Lankarani, H. M. 2016. A survey and comparison of several friction force models for dynamic analysis of multibody mechanical systems, Nonlinear Dynamics 86(3): 14071443. http://dx.doi.org/10.1007/s11071-016-2999-3.

S. Kilikevičius, A. Fedaravičius, V. Daukantienè, K. Liutkauskienè

\section{ANALYSIS ON CONVEYING OF MINIATURE AND MICROPARTS ON A PLATFORM SUBJECTED TO SINUSOIDAL DISPLACEMENT CYCLES WITH CONTROLLED DRY FRICTION}

\section{S u m m a r y}

This paper analyses the conveying process of miniature and microparts on a manipulation platform subjected to sinusoidal excitation in the horizontal direction when the effective coefficient of dry friction is being controlled. Hereby, the required dynamic directionality is achieved via the system asymmetry created by periodic alteration of the effective coefficient of dry friction between the micropart and the platform. A mathematical model of conveying process was developed applying the Dahl dynamic friction model. It was solved numerically and the influence of frictional properties, friction control and sinusoidal excitation parameters on the conveying process characteristics was determined. To test the theoretical observations in practice, an experimental setup for micropart conveying with controlled dry friction was created and build. Consistently with the modelling results, the experimental results demonstrated how the stable conveying velocity, direction and step size are controlled by regulating the parameters of the signals for sinusoidal displacement cycles and high frequency pulses in burst mode for dry friction control. The experimental findings qualitatively confirmed the trends observed by the modelling and showed that the proposed method can be applied in practice. The proposed method can be practically used in conveying, feeding, manipulation and assembly systems for miniature and microparts in the mechatronics, electronic and other industries.

Keywords: micropart, conveying, dynamic friction control, sinusoidal excitation, vibrations.

Received December 22, 2020 Accepted February 04, 2022 